\section{Aspectos climáticos em áreas de transmissão de hantavirose no Estado de São Paulo, Brasil}

\author{
Climatic aspects in hantavirus transmission areas in \\ São Paulo State, Brazil
}

\author{
${ }^{1}$ Faculdade de Ciências \\ Médicas, Universidade \\ Estadual de Campinas, \\ Campinas, Brasil. \\ 2 Instituto Adolfo Lutz, \\ São Paulo, Brasil. \\ ${ }_{3}$ Centro de Pesquisas \\ Meteorológicas e Climáticas \\ Aplicadas à Agricultura \\ Campinas, Brasil. \\ ${ }^{4}$ Centro de Vigilância \\ Epidemiológica, Secretaria \\ do Estado de Saúde de São \\ Paulo, São Paulo, Brasil. \\ Correspondência \\ M. R. Donalisio \\ Departamento de Medicina \\ Preventiva e Social, \\ Faculdade de Ciências \\ Médicas, Universidade \\ Estadual de Campinas. \\ Cidade Universitária, C. P. \\ 6111, Campinas, $S P$ \\ 13083-970, Brasil. \\ donalisi@fcm.unicamp.br
}

\begin{abstract}
The objective of this study was to investigate the spatial distribution of reported hantavirus cases in São Paulo State, Brazil $(n=80)$, from 1993 to 2005 and identify local climatic patterns during this period. Kernel point estimation of density was used to show the highest concentrations in Ribeirão Preto, São Carlos, Franca, Tupi Paulista, and Greater São Paulo. Increase in the number of cases during this period suggests disease dissemination even when considering increased diagnostic capacity and higher sensitivity of the health services. There was a marked seasonal variation in hantavirus in the cerrado (savannah) areas; the common pattern is a higher incidence in drier months as compared to mean levels in the last 40 years. These coincide with periods of high rodent food source levels in grains, sugarcane, and other crops. Harvesting and storing grains increases human exposure to rodents. Climatic indicators together with ecological variables can be local transmission risk markers and should receive more attention in epidemiological monitoring and control of the disease.
\end{abstract}

Hantavirus Pulmonary Syndrome; Spatial Analysis; Climate

\author{
Maria Rita Donalisio 1 \\ Cíntia Honório Vasconcelos 1 \\ Luiz Eloy Pereira 2 \\ Ana Maria H. Ávila ${ }^{3}$ \\ Giselda Katz 4
}

\section{Introdução}

Na natureza, o hantavírus causa infecção assintomática e persistente em roedores transmitindo-se a humanos por meio de inalação de aerossóis, a partir de urina, fezes e saliva destes animais, ou por contato direto do vírus em pele lesada, escoriações ou mordeduras de ratos 1,2. O hantavírus pertence à família Bunyaviridae, cujas linhagens correspondem aos locais onde foram isolados 1 .

A hantavirose pulmonar é uma antropozoonose notificada pela primeira vez no Brasil em área rural do Município de Juquitiba, Estado de São Paulo, com a ocorrência de três casos em 1993 3. Até dezembro de 2005, 687 casos foram notificados no país, em todas as regiões, sendo oitenta confirmados e autóctones no Estado de São Paulo (11,6\%), segundo o Sistema de Vigilância Epidemiológica da Secretaria de Estado de Saúde de São Paulo (SES-SP; http:/ / www.cve. saude.sp.gov.br/htm/zoo/hanta_dados.html).

Os dados da doença no Brasil indicam que mais de $50 \%$ dos casos ocorrem em indivíduos com atividades agropecuárias, sendo possivelmente a zona rural o principal local de transmissão 1,2 .

No Estado de São Paulo circulam dois vírus causadores da hantavirose humana, o Araraquara e o Juquitiba. O primeiro é associado ao $\mathrm{Bo}$ lomys lasiurus, roedor mais abundante nas áreas de cerrado brasileiro e caatinga do Nordeste 
brasileiro, e acredita-se que é o mais importante reservatório do hantavírus no Estado de São Paulo; enquanto o vírus Juquitiba é associado ao Oligoryzomys nigripes, principal agente etiológico da hantavirose nas áreas de Mata Atlântica e Mata de Araucária ${ }^{4}$. Em vários estudos sobre a dinâmica de roedores em áreas de cerrado, registrou-se que o $B$. lasiurus foi uma das espécies capturadas em maior densidade 4,5,6,7 .

Observa-se que cada variante viral está envolvida com uma única espécie de roedor como seu principal reservatório, sugerindo a distribuição geográfica focal destas variantes virais ? O risco da infecção pelo hantavírus está diretamente relacionado com a densidade populacional de roedores, bem como com a probabilidade de contato destes animais infectados com populações humanas 7,8,9. Assim, a cadeia de transmissão é influenciada pelo contexto ambiental, ecológico e das relações do homem com este ambiente, sendo que estes componentes se apresentam de forma diferente em várias regiões onde se descreve a doença nas Américas $7,10,11,12,13$.

Pouco se conhece sobre as condições ecológicas, as circunstâncias da transmissão em humanos e as especificidades dos ecossistemas que facilitam a propagação dos reservatórios e a circulação da infecção 7,8,10,12. A distribuição regional do hospedeiro e do patógeno está relacionada com fatores ambientais climáticos e vegetação, o que reforça a necessidade de estudo sobre os distintos biomas para a compreensão dos padrões espaciais e temporais de ocorrência de casos 7,8.

Alguns autores chamam a atenção para o papel do clima interferindo na dinâmica populacional de roedores devido a alterações ambientais que interferem na disponibilidade de alimentos a estas espécies reservatório 12,14. O surto de síndrome hantavirose pulmonar que ocorreu em 1993 no sudoeste dos Estados Unidos foi associado ao fenômeno El Niño (19921993) pelo aumento da precipitação e particularmente pelo pico observado no meio da estação seca 12 .

Técnicas de geoprocessamento, análise espacial e dados climáticos podem contribuir para melhorar a compreensão dos diversos componentes ambientais e antrópicos envolvidos na dinâmica da produção e transmissão da hantavirose 12,15 .

Este trabalho tem como objetivo investigar a distribuição espacial dos casos notificados de hantavirose no Estado de São Paulo, de 1993 a 2005, e identificar os padrões climáticos locais no período provável de infecção.

\section{Método}

Estudo ecológico exploratório sobre as áreas de ocorrência de hantavirose no Estado de São Paulo, Brasil, de 1993 a 2005. Os oitenta casos autóctones confirmados no estado pelo Sistema de Vigilância Epidemiológica foram localizados no mapa utilizando-se o centróide do município de ocorrência confirmado na ocasião da investigação epidemiológica. Por se tratar de mapa em escala pequena, optou-se pela utilização do centróide para homogeneizar a identificação do município no mapa do estado, embora a endemia seja eminentemente rural, portanto afastada do centróide.

$$
\hat{\lambda_{\tau}}(s)=\sum_{h_{j}<\tau} \frac{3}{\pi \tau^{2}}\left(1-\frac{h_{j}^{2}}{\tau^{2}}\right)^{2}
$$

\section{Análise espacial}

Foram identificadas as regiões do estado com maior concentração de casos por meio do estimador de densidade de kernel, utilizando-se a equação quadrática acima 16 :

Em que $\lambda$ é o resultado da função densidade de casos, $\tau$ é o raio de abrangência de cada área na superfície do mapa de estudo e h a distância entre o ponto central e cada ponto dentro da área delimitada por $\tau$. O raio de abrangência $(\tau)$ escolhido foi de $100 \mathrm{~km}$, permitindo identificar aglomerados em microrregiões com características ambientais semelhantes (clima e vegetação). Os casos foram distribuídos em três períodos distintos para a visualização da progressão da incidência da hantavirose no estado: 1993 a 1996, 1997 a 2000 e 2001 a 2005. Com base nos mapas de densidade foram identificadas quatro regiões com maior concentração de casos: (1) litorânea, composta por Juquitiba até Mogi das Cruzes, região predominantemente de Mata Atlântica com relevo acidentado e com dez casos; (2) São Carlos com 14 casos; (3) Ribeirão Preto com 40 notificações no período de estudo; e (4) região de Tupi Paulista e Adamantina com dez casos, localizada no oeste do estado.

Foram analisadas apenas as áreas de aglomeração de casos (Figura 1), constituindo-se as regiões de estudo, onde se identificaram 64 locais prováveis de infecção ( $80 \%$ do total), nas regiões de cerrado no norte e oeste do estado, que correspondem às regiões de Tupi Paulista e Ribeirão Preto, além do centro, em São Carlos. Nessas áreas foi notificada a presença de reservatórios e vírus comuns envolvidos com os casos de hantavirose, o B. lasiurus e o vírus Araraquara $4,7,16,17$. Para maior detalhamento do ocorrido na região de Ribeirão Preto, esta foi separada em 
três microrregiões para análise: Franca, Cássia dos Coqueiros e Ribeirão Preto.

Casos isolados em outras regiões do estado $(6,7,5 \%)$ não foram analisados, tampouco os que ocorreram na região de Mata Atlântica (10, 12,8\%) (Juquitiba, Mogi das Cruzes, Embu, Barra do Turvo) por fazerem parte de ecossistema de Mata Atlântica, onde o reservatório envolvido na transmissão é o $O$. nigripes e o vírus circulante é o Juquitiba.

\section{Análise temporal (pluviosidade)}

As áreas foram classificadas em padrões climáticos, segundo a classificação de Koeppen (Figura 1) 18 .

Na região litorânea de Mata Atlântica, o clima caracteriza-se como do tipo Af, tropical chuvoso, sem estação seca, com a precipitação média do mês mais seco superior a $60 \mathrm{~mm}$ pela classificação de Koeppen ${ }^{18}$. As regiões de Ribeirão Preto e São Carlos são as áreas de maior incidência da doença, onde se registra intensa produção agrícola, principalmente da cana-de-açúcar na região de Ribeirão Preto. Em São Carlos predomina o clima Cwa, tropical de altitude com chuvas no verão e seca no inverno, com temperatura média no inverno menor que $22^{\circ} \mathrm{C}$. Nas regiões de Ribeirão Preto e Tupi prevalece o clima Aw, tropical chuvoso com inverno seco e mês mais frio com temperatura média superior a $18^{\circ} \mathrm{C}$. O mês mais seco tem precipitação inferior a $60 \mathrm{~mm}$ e com período chuvoso que se atrasa para o outono 18 .

Foi analisada a distribuição mensal dos casos no período de 1993 a 2005 nas áreas de cerrado e clima tropical chuvoso com inverno seco (Aw) no Estado de São Paulo, utilizando-se a data dos primeiros sintomas como referência temporal.

Medidas de precipitação pluviométrica em milímetros (mm) foram obtidas de estações instaladas nos municípios de referência ou nas proximidades das áreas de transmissão, disponibili-
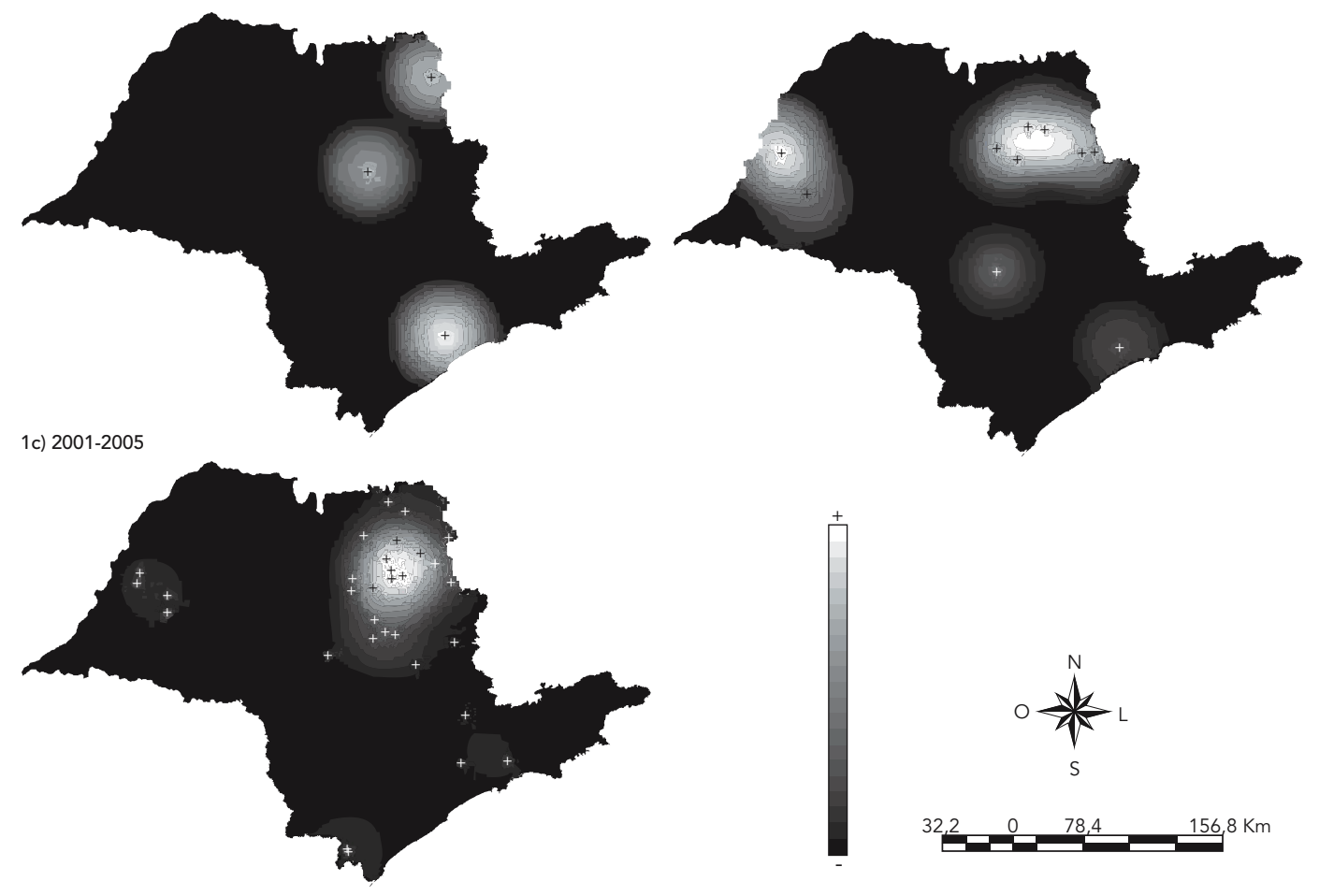
zadas pelo Instituto Agronômico de Campinas, do Departamento de Águas e Energia Elétrica (DAEE) e do Ministério da Agricultura (Agritempo) divulgadas pela SES-SP 19. Foram calculadas as médias de precipitação pluviométrica mensais dos anos de 1961 a 2005 e calculados os desvios padrão acima e abaixo destas médias. Foram comparadas as medidas locais de pluviosidade dos meses e anos de ocorrência de casos em cada região com as médias e dispersão dos dados de décadas anteriores.

A pluviosidade (mm) foi privilegiada na análise devido à sua influência direta na composição e vigor da vegetação, reconhecido indicador de disponibilidade de alimentos favoráveis à reprodução e sobrevivência de roedores silvestres 5,7,8,14.

Para a elaboração dos mapas e estimadores de densidade de pontos, foi utilizado o programa SPRING versão 4.01 Beta (Instituto Nacional de Pesquisas Espaciais, São José dos Campos, Brasil) e para as planilhas e gráficos utilizou-se o programa Excel 2002 (Microsoft Corp., Estados Unidos).

\section{Resultados}

Observa-se o progressivo aumento na notificação de casos e uma crescente dispersão das ocorrências no território do estado, chegando a oitenta casos autóctones confirmados até dezembro de 2005. Desde a primeira notificação da hantavirose em 1993 até o ano de 1996 foram identificados cinco casos, de 1997 a 2000, 16 casos e de 2001 a 2005 foram registrados 59 pacientes com hantavirose no Estado de São Paulo.

A Figura 3 mostra a distribuição mensal dos casos ocorridos no estado, segundo a data dos primeiros sintomas, indicando um padrão sazonal de maior ocorrência nos meses secos, de outono e inverno. As médias mensais de pluviosidade das quatro regiões de estudo apresentam tendência inversa da ocorrência dos casos, sendo notificados $80 \%$ entre abril e setembro e $20 \%$ entre outubro e março, com maior concentração nas áreas de cerrado com clima Aw (Figuras 2 e 3).

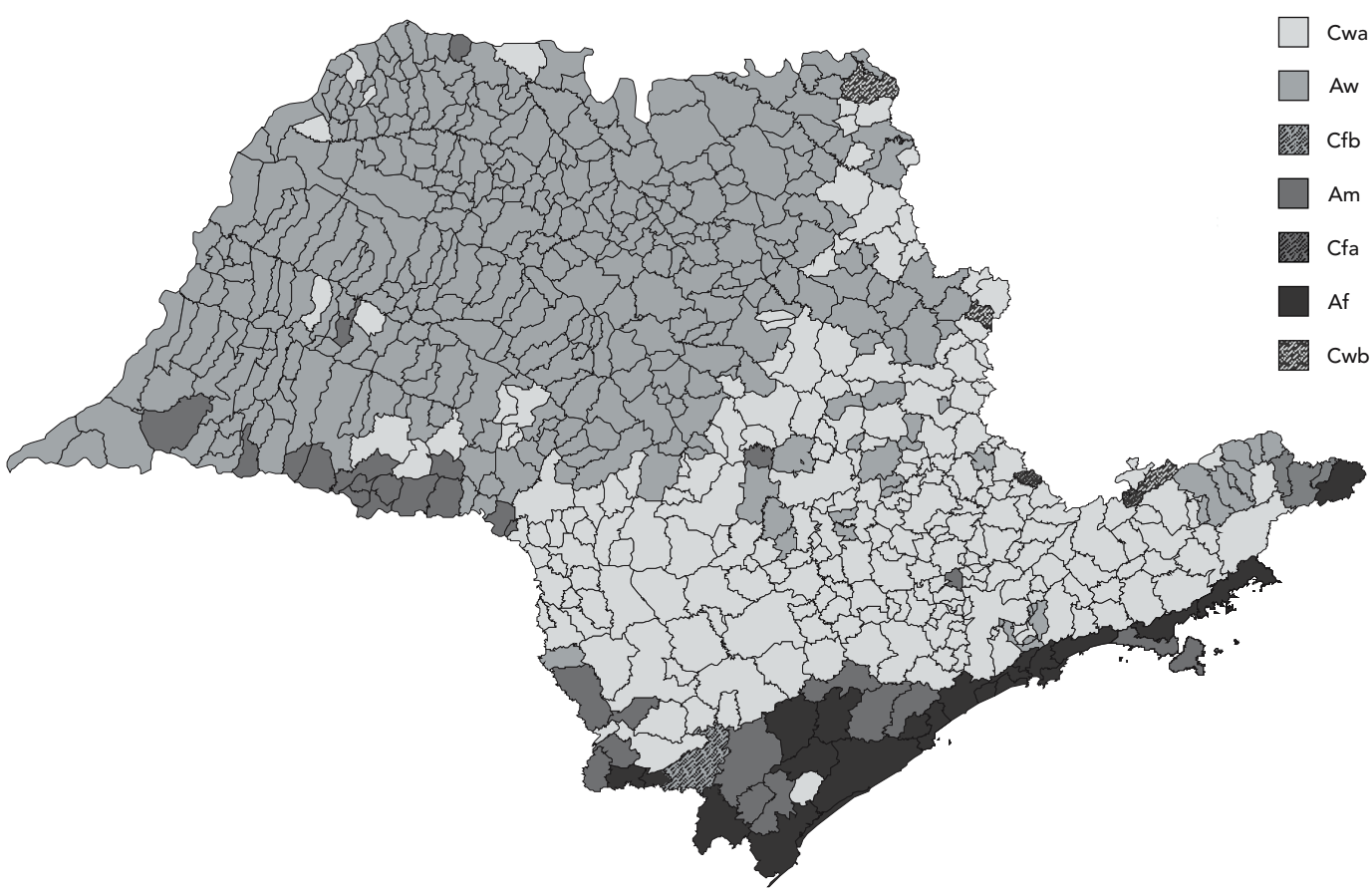

Nota: região Aw corresponde em parte a regiões de vegetação de cerrado 18 . 


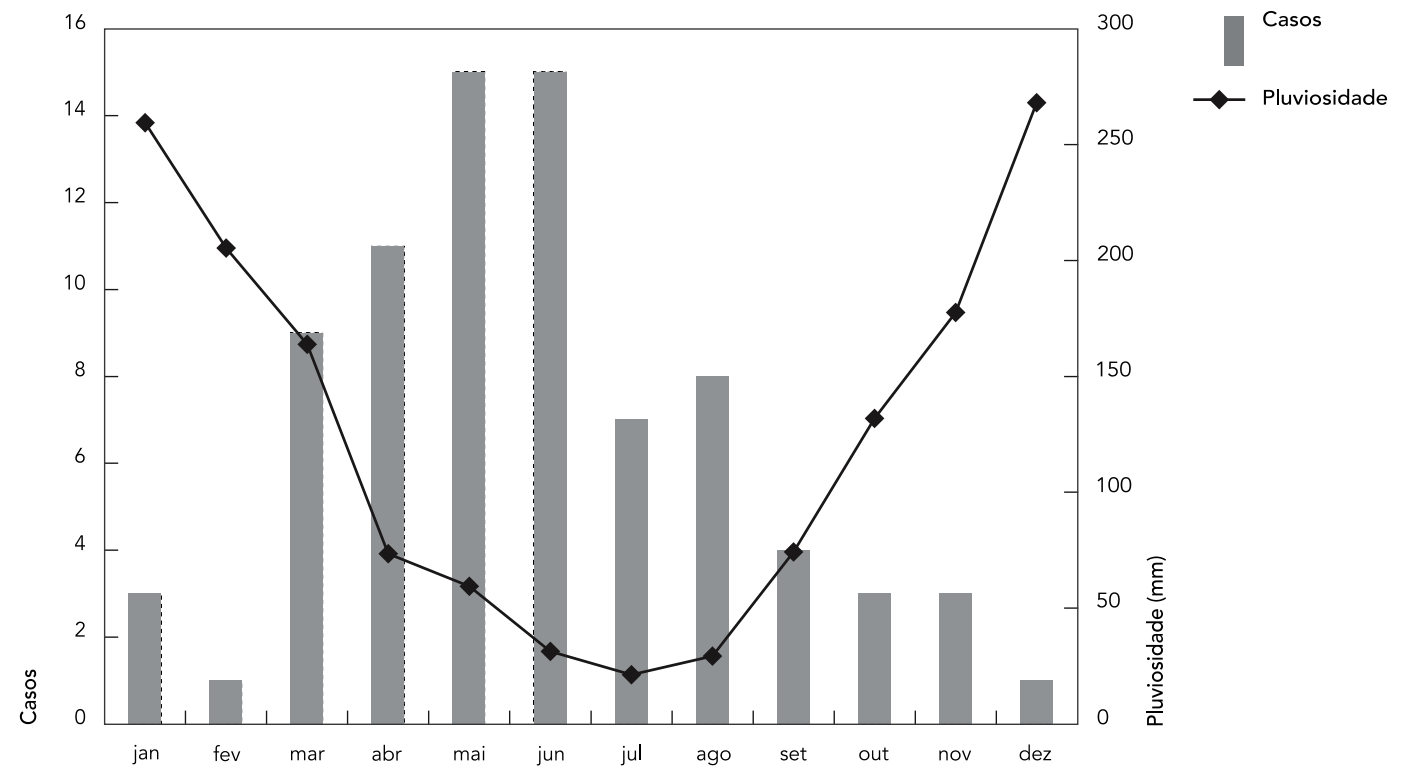

Nota: para cálculos foram utilizados dados de pluviosidade (mm) de 1960 a 2005

Fonte: Ministério da Agricultura ${ }^{19}$ e Secretaria de Estado de Saúde de São Paulo.

Os mapas apresentados na Figura 1 indicam a intensidade de ocorrência da doença utilizando-se o estimador de densidade de kernel. Identificam-se quatro áreas onde há maior concentração de casos nos vários períodos de estudo: Tupi Paulista (10 casos), Ribeirão Preto (40), São Carlos (14) e Litorânea (10).

Ao relacionar-se os meses e anos da infecção com os dados de pluviosidade, procurou-se analisar características ambientais particulares de cada microrregião de estudo.

Os dados locais de precipitação pluviométrica em mm são apresentados nas Figuras 4, 5 e 6. As curvas mostram as médias mensais dos últimos quarenta anos e um desvio-padrão acima e abaixo destas médias e a pluviosidade média dos meses em anos de ocorrência dos casos. Os gráficos mostram tendência comum, isto é, a curva da precipitação pluviométrica na ocasião da infecção aproxima-se ou coincide com a curva da média menos um desvio-padrão, indicando que se trata de um período mais seco que as médias de chuva das últimas décadas $(68 \%$ de confiabilidade) naquela região e mês. Este padrão foi constatado com os dados pluviométricos e ocorrência de casos na região de Franca,
Tupi Paulista, Cássia dos Coqueiros e Ribeirão Preto (neste último, dados não apresentados). Estes períodos foram antecedidos por períodos de maior pluviosidade comparada às médias das décadas anteriores.

\section{Discussão}

O aumento do número de casos nos vários períodos de estudo sugere disseminação da doença no estado. Porém há que se considerar o aumento da capacidade diagnóstica e maior sensibilidade do sistema de saúde, particularmente em áreas onde já houve registro de casos.

Neste estudo, o aspecto climático privilegiado foi a pluviosidade (mm). A temperatura não foi analisada como variável determinante de alterações na dinâmica populacional de roedores. As temperaturas médias, mínimas e máximas das regiões de estudo não se diferenciaram significativamente das registradas nos anos de notificação dos casos em cada região de estudo, além de não alcançarem limiares que poderiam ter comprometido a sobrevivência de espécies de roedores associadas com a hantavirose. 
Pluviosidade média $(\mathrm{mm}) \pm 1$ desvio-padrão * e pluviosidade nos anos e meses de ocorrência de casos ** de hantavirose na região de Franca, São Paulo, Brasil, 2003 e 2004

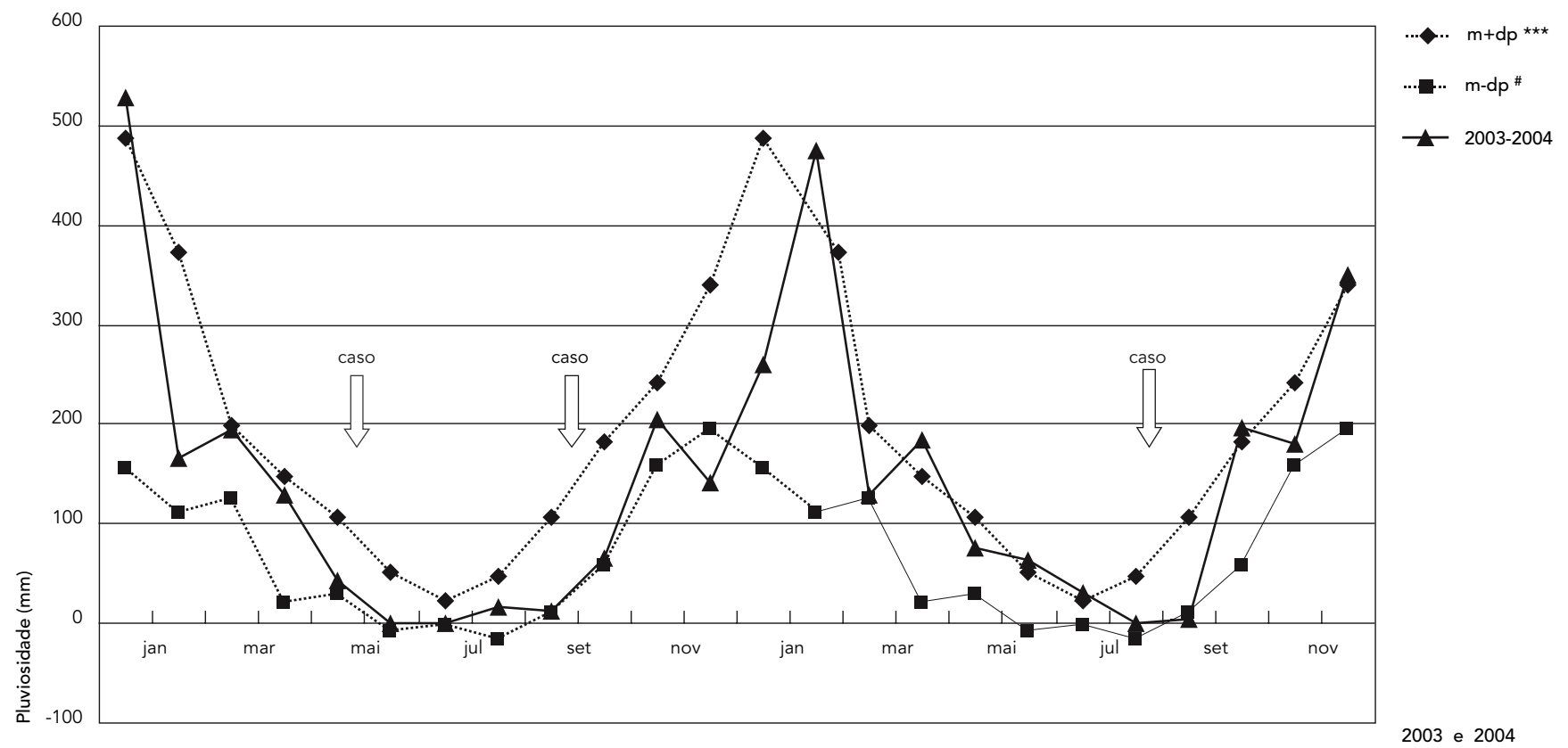

* Para cálculos utilizaram-se dados de pluviosidade (mm) de 1960 a 2005;

** Ocorrência dos casos = data dos primeiros sintomas;

*** $\mathrm{m}+\mathrm{dp}=$ média +1 desvio-padrão da pluviosidade;

\# m-dp = média - 1 desvio-padrão da pluviosidade.

Fonte: Ministério da Agricultura 19 e Secretaria de Estado de Saúde de São Paulo.

As regiões de clima Aw (tropical chuvoso com inverno seco), particularmente o cerrado no Estado de São Paulo, sofreram processo de intensa modificação devido à expansão da agricultura. Essas modificações podem proporcionar excesso de alimento em períodos que naturalmente estariam escassos, influenciando a composição de espécies de roedores. Um habitat antropizado suporta menor diversidade de espécies, pois promove intensa competição e favorece espécies generalistas e oportunistas 14,20,21. Vários estudos têm verificado que o $B$. lasiurus é uma das espécies mais encontradas em ambiente de cerrado, por ser possivelmente uma das espécies com maior possibilidade de adaptação e competição em ambientes modificados 20. Esse roedor tem sido relacionado com a transmissão de hantavirose nas áreas de cerrado no Estado de São Paulo 4.

O crescimento e reprodução do B. lasiurus mostram tendência sazonal, variando em di- ferentes ecossistemas estudados. A densidade varia temporalmente com a disponibilidade de alimentos e ninhos, e espacialmente com a estrutura do habitat e tipo de vegetação 5,7,14,22.

O período reprodutivo desses roedores silvestres começa simultaneamente com o início das chuvas em outubro, atingindo seu pico em janeiro. Os filhotes nascidos nesse período tornam-se adultos a partir de março. Os roedores adultos apresentam maior soroprevalência de anticorpos contra o hantavírus, devido à crescente probabilidade de exposição ao vírus, contraído em brigas e disputas, fato observado em diversas espécies associadas à hantavirose 9 .

O período de maior densidade do B. lasiurus, em regiões de cerrado, corresponde ao período de seca (maio a outubro) que também coincide com as colheitas e com o aumento da disponibilidade de sementes no solo 5,6,7, especialmente do capim braquiária 4,13. A disponibilidade de alimento pode ser um dos principais fatores que 
Pluviosidade média $(\mathrm{mm}) \pm 1$ desvio-padrão * e pluviosidade nos anos e meses de ocorrência de casos ** de hantavirose na região de Cássia dos Coqueiros, São Paulo, Brasil, 1999 a 2003.

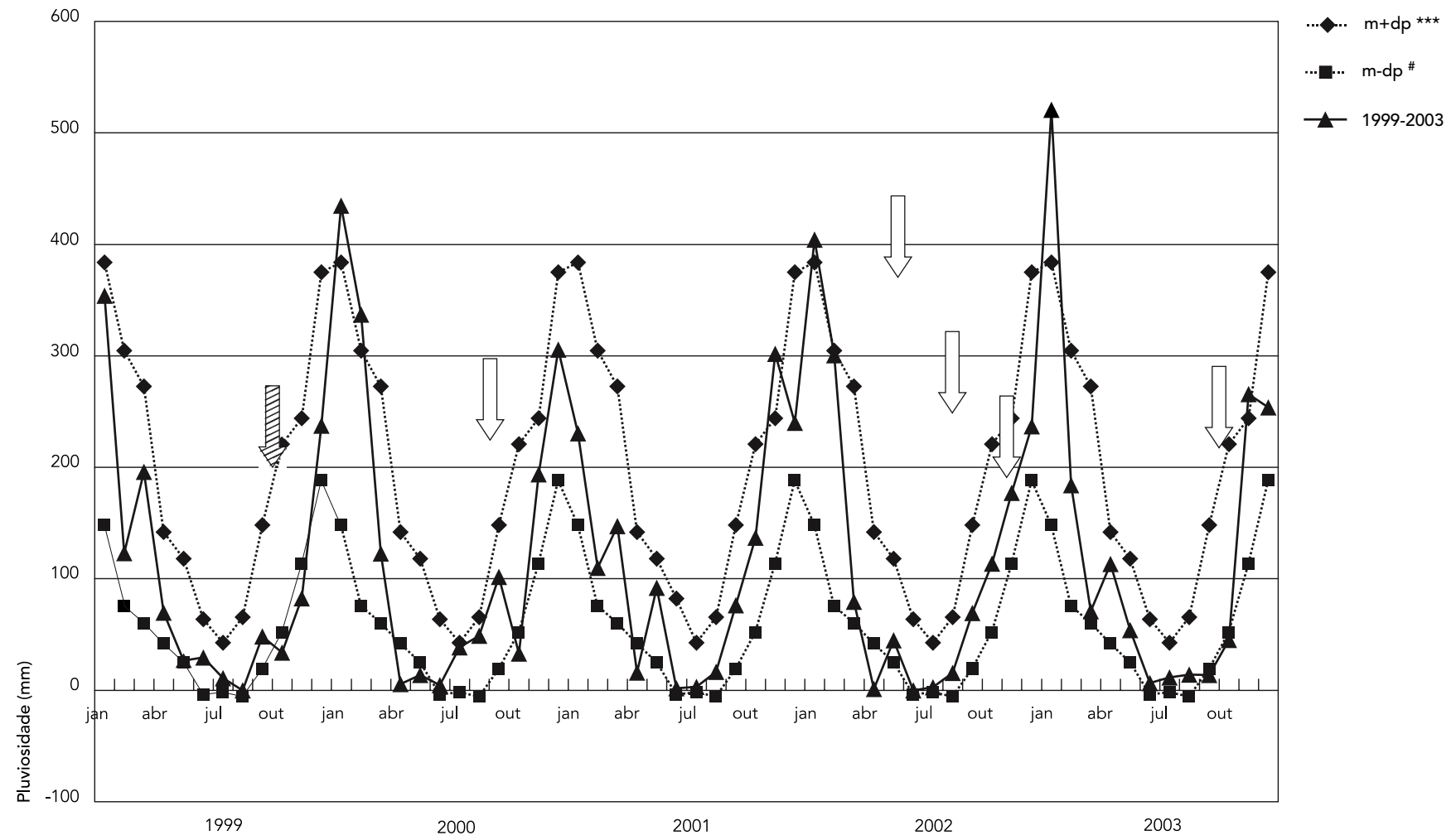

* Para cálculos utilizaram-se dados de pluviosidade de 1960 a 2005;

** Ocorrência dos casos = data dos primeiros sintomas;

*** $\mathrm{m}+\mathrm{dp}=$ média +1 desvio-padrão da pluviosidade;

\# m-dp = média - 1 desvio-padrão da pluviosidade.

Nota: flecha tracejada significa dois casos no mesmo mês.

Fonte: Ministério da Agricultura 19 e Secretaria de Estado de Saúde de São Paulo.

explicam a sobrevivência e circulação desses roedores. Entre as principais culturas relacionadas com a que atua dessa forma, destacam-se milho, soja, arroz, trigo, sorgo, aveia, capim braquiária, capim colonião, cana-de-açúcar, batata doce, mandioca, o plantio de pinheiro (Pinus sp.) e eucalipto (Eucaliptus sp.). No caso da cultura de cana-de-açúcar, o período de seca coincide com a colheita da cana, antecedido pelas queimadas as quais provocam êxodo de roedores para as margens da cultura, bordas de matas e locais ocupados pelo homem.

Possivelmente, as chuvas têm influência indireta na sobrevivência e reprodução de roedores, propiciando o aumento de frutos, sementes e insetos, fontes de alimentos das espécies. Pois, além do adensamento da população de roedores comum na época seca do ano, a transmissão da síndrome hantavirose pulmonar ao homem está associada a fatores e fenômenos ambientais que forçam os deslocamentos da população de roedores. As inundações, queimadas e desmatamentos são os principais fatores que desalojam as colônias de roedores e as obrigam procurar novos ambientes, aumentando o risco de exposição do homem pela invasão de roedores nas áreas habitadas 5,10,23.

Neste estudo, verificou-se marcada sazonalidade dos casos nas áreas de cerrado do Estado de São Paulo, incidindo com freqüência em 
Pluviosidade média $(\mathrm{mm}) \pm 1$ desvio-padrão * e pluviosidade nos anos e meses de ocorrência de casos ** de hantavirose na região de Tupi Paulista, São Paulo, Brasil, 1998 a 1999.

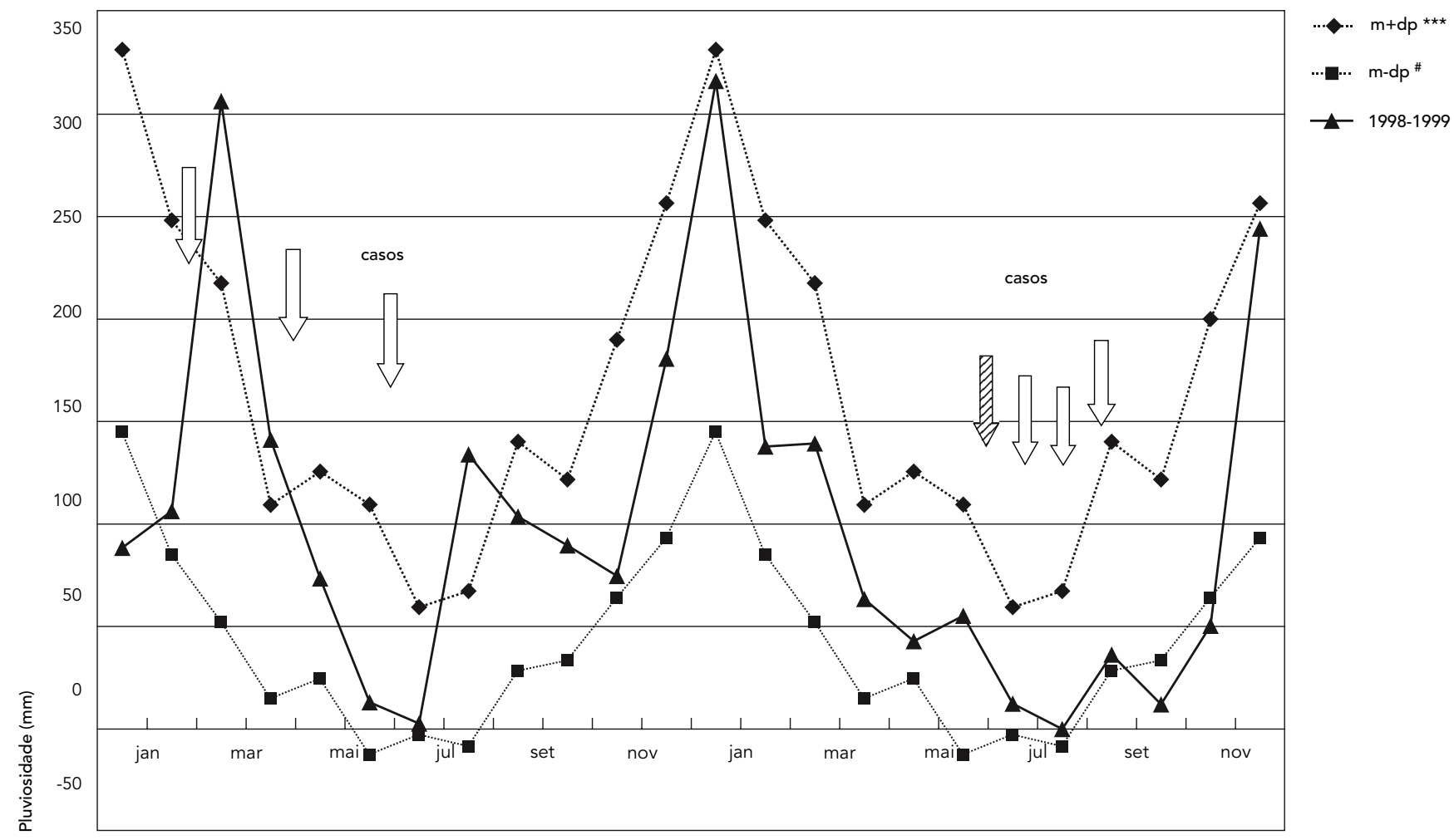

\footnotetext{
* Para cálculos utilizaram-se dados de pluviosidade de 1960 a 2003;

** Ocorrência dos casos = data dos primeiros sintomas;

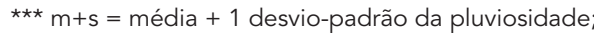

\# m-s = média - 1 desvio-padrão da pluviosidade.

Nota: flecha tracejada significa 2 casos no mesmo mês.

Fonte: Ministério da Agricultura 19 e Secretaria de Estado de Saúde de São Paulo.

meses particularmente secos, comparados com as médias dos últimos quarenta anos. O estudo do período médio de incubação como referência temporal de infecção, procurou aproximar a ocasião provável de infecção com os dados pluviométricos.

Outras variáveis interferindo no risco de transmissão necessitam ser melhor investigadas, como os padrões de contato com os roedores e os tipos de culturas, o armazenamento de grãos, o processo de trabalho e as condições de moradia.

Embora o estudo ecológico não possa sustentar argumentos sobre a associação entre o regime de chuvas e o risco de infecção, evidencia uma tendência, um padrão de pluviosidade na ocasião da transmissão. Esta é uma análise exploratória da associação entre chuva e hantavirose, que pode ser aprofundada utilizando-se técnicas de modelagem que permitem estimar o efeito da chuva e o padrão de sazonalidade. Assim, essas circunstâncias climáticas moduladas por um conjunto de outras variáveis podem se constituir em marcadores de risco de transmissão local a serem valorizados nas ações de vigilância e controle da doença. 


\section{Resumo}

O objetivo deste trabalho foi investigar a distribuição espacial dos casos notificados de hantavirose $(n=80)$ no Estado de São Paulo, Brasil, de 1993 a 2005, e identificar os padrões climáticos locais nos anos de registro da doença. Foi utilizado o estimador de densidade de pontos de kernel que apontou maior concentração nas regiões de Ribeirão Preto, São Carlos, Franca, Tupi Paulista e Grande São Paulo. O aumento do número de casos no período sugere disseminação da doença, embora se considere o aumento da capacidade diagnóstica e maior sensibilidade do sistema de saúde. Registrou-se marcada sazonalidade da hantavirose nas áreas de cerrado, com padrão comum, isto é, maior incidência em meses com baixa pluviosidade, comparados com médias dos últimos quarenta anos. Esses períodos coincidem com épocas de maior disponibilidade de alimentos a roedores em cultura de grãos, cana e outros. A colheita e armazenamento desses grãos aumentam a exposição de populações humanas aos roedores. Indicadores climáticos em conjunto com variáveis ecológicas podem compor marcadores de risco de transmissão local a serem valorizados nas ações de vigilância epidemiológica e controle da doença.

Síndrome Pulmonar por Hantavirus; Análise Espacial; Clima

\section{Contribuição}

M. R. Donalisio e C. H. Vasconcelos participaram da concepção do trabalho, análise dos dados e redação do texto. A. M. H. Ávila participou da coleta e análise dos dados e revisão do texto. L. E. Pereira e G. Katz participaram da discussão e análise dos resultados e da revisão do manuscrito.

\section{Agradecimentos}

A Celso Stephan (Laboratório de Análise Espacial de Dados Epidemiológicos, Universidade Estadual de Campinas). Este trabalho obteve financiamento do Departamento de Ciência e Tecnologia, Ministério da Saúde e do Conselho Nacional de Desenvolvimento Científico e Tecnológico.

\section{Referências}

1. Ferreira MS. Hantavirose. Rev Soc Bras Med Trop 2003; 36:81-96.

2. Secretaria de Vigilância em Saúde, Ministério da Saúde. Aspectos epidemiológicos da infecção e da patogenicidade por hantavirose no Brasil (2004). Boletim SVS 2005; 5:2-5.

3. Iverson LB, Travassos APA, Rosa MDB, Lomar AV, Sasaki MGM, Leduc JM. Infecção humana por hantavírus no Sul e Sudeste do Brasil. Rev Assoc Med Bras 1994; 40:85-92.

4. Susuki A, Bisordi I, Levis S, Garcia J, Pereira LE, Souza RP, et al. Identifying rodent hantavirus reservoir, Brazil. Emerg Infect Dis 2004; 10:2100-7.

5. Alho CRJ, Pereira LA. Population ecology of cerrado rodent community in central Brazil. Rev Bras Biol 1985; 45:597-607.

6. Vieira MV. Dynamics of a rodent assemblage in a cerrado of Southeast Brazil. Rev Bras Biol 1997; 57:99-107.
7. Pereira LE. Estudo epidemiológico de hantavírus em roedores das regiões da Mata Atlântica e cerrado do Brasil [Tese de Doutorado]. São Paulo: Coordenadoria de Controle de Doenças, Secretaria de Estado de Saúde de São Paulo; 2006.

8. Mills JN, Childs JE. Ecologic studies of rodent reservoirs: their relevance for human health. Emerg Infect Dis 1998; 4:529-37.

9. Childs JE, Krebs JW, Ksiasek TG, Maupin GO, Gage KL, Rollin PE, et al. A household based case-control study of environmental factors associated with hantavirus pulmonary syndrome in the southwestern United States. Am J Trop Med Hyg 1995; 52:393-7.

10. Busch M, Cavia R, Carbajo AE, Bellomo C, Gonzalez-Capria S, Padula P. Spatial and temporal analysis of hantavirus pulmonary syndrome in Buenos Aires Province and its relation to rodent distribution agricultural and demographic variables. Trop Med Int Health 2004; 9:508-19. 
11. Noemi P. Hantavirus pulmonary syndrome in Latin America. Curr Opin Infect Dis 2004; 17:427-31.

12. Engelthaler DM, Mosley DG, Cheek JE, Levy CE, Kenneth K, Komatsu PE, et al. Climatic and environment patterns associated with hantavirus pulmonary syndrome, Four Corners Region, United States. Emerg Infect Dis 1999; 5:87-94.

13. Katz G, Willians RJ, Burt MS, Souza LT, Pereira LE, Mills JN, et al. Hantavirus pulmonary syndrome in the estate of São Paulo, Brazil 1993-1998. Vector Borne Zoonotic Dis 2001; 1:181-90.

14. Francisco AL, Magnusson WE, Sanaiotti TM. Variation in growth and reproduction of Bolomys lasiurus (Rodentia: Muridae) in Amazonian savanna. J Trop Ecol 1995; 11:419-28.

15. Henkes WE, Barcelos C. Ecologia da paisagem da hantavirose no Estado do Rio Grande do Sul. Rev Soc Bras Med Trop 2004; 37:505-7.

16. Bailey TC, Gatrell AC. A Interactive spatial data analysis. London: Prentice Hall; 1995.

17. Figueiredo LT, Campos GM, Rodrigues FB. Hantavirus pulmonary and cardiovascular syndrome: epidemiology, clinical presentation, laboratory diagnosis and management aspects. Rev Soc Bras Med Trop 2001; 34:13-34.
18. Koeppen W. Climatología. México DF: Fondo de Cultura Económica; 1948.

19. Ministério da Agricultura. Agritempo. http://www. agritempo.gov.br (acessado em 05/Mai/2006).

20. Alho CJR. Small mammal populations of Brazilian cerrado: the dependence of abundance and diversity on habitat complexity. Rev Bras Biol 1981; 41:223-30.

21. Mills JN. Biodiversity loss and emerging infectious disease: an example from the rodent-borne hemorrhagic fevers. Biodiversity 2006; 7:9-17.

22. Ghizoni IR, Layme VMG, Lima AP, Magnusson WE. Spatially explicit population dynamics in a declining population of tropical rodent Bolomys lasiurus. J Mammal 2005; 86:677-82.

23. Briani DC, Palma ART, Vieira EM, Henriques RPB. Post fire succession of small mammals in the cerrado of central Brazil. Biodivers Conserv 2004; 13:1023-37.

Recebido em 16/Ago/2006

Versão final reapresentada em 28/Set/2007

Aprovado em 01/Out/2007 